Challenges in investigation of diabetes-related aviation fatalitiesan analysis of 1491 subsequent aviation fatalities in USA during 2011-2016

\author{
Junttila, Ilkka S.
}

2018-11

Junttila , I S , Vuorio , A , Budowle , B , Laukkala , T \& Sajantila , A 2018 , ' Challenges in investigation of diabetes-related aviation fatalitiesan analysis of 1491 subsequent aviation fatalities in USA during 2011-2016 ' , International Journal of Legal Medicine, vol. 132 , no. 6 , pp. 1713-1718 . https://doi.org/10.1007/s00414-018-1879-4

http://hdl.handle.net/10138/260565

https://doi.org/10.1007/s00414-018-1879-4

publishedVersion

Downloaded from Helda, University of Helsinki institutional repository.

This is an electronic reprint of the original article.

This reprint may differ from the original in pagination and typographic detail.

Please cite the original version. 


\title{
Challenges in investigation of diabetes-related aviation fatalities-an analysis of 1491 subsequent aviation fatalities in USA during 2011-2016
}

\author{
Ilkka S. Junttila ${ }^{1,2} \cdot$ Alpo Vuorio $^{3,4} \cdot$ Bruce Budowle $^{5} \cdot$ Tanja Laukkala $^{6} \cdot$ Antti Sajantila $^{4}$
}

Received: 1 February 2018 / Accepted: 15 June 2018 / Published online: 4 July 2018

(C) Springer-Verlag GmbH Germany, part of Springer Nature 2018

\begin{abstract}
Diabetes mellitus (DM) could cause pilot incapacitation and result in aviation fatalities. The mechanisms could be directly as a consequence of acute hypoglycemia/subacute diabetic ketoacidosis (DKA) or indirectly as an acute cardiovascular event by contributing to the development of atherosclerosis in coronary or carotid and cerebral arteries. In this study, DM-related fatal flight accidents in the US National Transport Bureau's database between years 2011-2016 were analyzed with special emphasis on postmortem (PM) glucose levels and correlation of toxicological reports with anamnestic information on DM. Additionally, autopsy results on coronary arteries were reviewed. In 43 out of $1491(\sim 3 \%)$ fatal accidents pilots had DM. Postmortem glucose or glycated hemoglobin percentage (Hb1Ac) was measured in 12 of the 43 cases; while antidiabetic medication was found in 14 of the cases (only two of the cases had both glucose measurements and medication). With the increasing prevalence of DM, a possibility of pilot incapacitation due to DM or complications of DM should be actively studied, even if no anamnestic information of DM was available. While PM hypoglycemia is difficult to assess, we propose a systematic investigation based on measurement of glucose, $\mathrm{Hb} 1 \mathrm{Ac} \%$, and ketone bodies, and documentation of atherosclerotic lesions in major arteries to identify or rule out DM as a cause of pilot incapacitation.
\end{abstract}

Keywords Pilot incapacitation · Fatal flight accident · Accident investigation · Diabetes · Glucose measurement · Diabetic ketoacidose $\cdot$ Hypoglycemia $\cdot$ Hyperglycemia

\section{Abbreviations}

FAA Federal aviation administration

AME Aviation medical examiner

ICAO International civil aviation organization

CAA Civil aviation authority

Ilkka S. Junttila and Alpo Vuorio contributed equally to this work.

Antti Sajantila

antti.sajantila@helsinki.fi

1 Faculty of Medicine and Life Sciences, University of Tampere, Tampere, Finland

2 Fimlab Laboratories, Tampere, Finland

3 Mehiläinen Airport Health Centre, Vantaa, Finland

4 Department of Forensic Medicine, University of Helsinki, Helsinki, Finland

5 Center for Human Identification, University of North Texas Health Science Center, 3500 Camp, Bowie Blvd., Fort Worth, TX 76107, USA

6 Mehiläinen Kielotie Health Centre, Vantaa, Finland
NTSB National transportation safety board

DKA Diabetic ketoacidosis

HbA1c Glycated hemoglobin

AMI Acute myocardial infarction

CNS Central nervous system

PM Postmortem

CO Carbon monoxide

\section{Introduction}

Roughly 30.3 million adults in the USA have diabetes (diabetes mellitus, DM) and the overall prevalence of DM is $9.4 \%$. Strikingly, of these individuals, 7.2 million are not aware they have DM ( $\sim 24 \%$ of all diabetics) (http://www.diabetes.org/ diabetes-basics/statistics/). In aviation, DM does not necessarily prevent a pilot from obtaining a license to fly. In the UK, the UK Civil Aviation Authority (CAA) may permit, according to recent practice, a class I medical certificate (required for all pilots involved in commercial aviation) to commercial pilots with type I DM upon careful monitoring of 
blood glucose level [1]. In contrast in the USA, insulin-treated DM remains an absolute disqualifying condition for obtaining a class I medical certificate; however, private pilots may obtain a specific waiver to obtain a certificate to fly [2]. As for aviation safety, since $\sim 24 \%$ of people with DM are unaware of their disease (http://www.diabetes.org/diabetes-basics/ statistics/), screening of pilots for undiagnosed DM should be a requisite. The screening necessity is of particular importance given an increasing aging of the general population (note that prevalence of DM in individuals 65 years and older is $25.2 \%$; http://www.diabetes.org/ diabetes-basics/statistics/) and that in the USA currently only very limited checkups for aging pilots are required [3].

Pilot incapacitation due to DM-related causes may occur gradually or suddenly. Typical forms of sudden incapacitation are hypoglycemia, in which loss of consciousness is due to lack of glucose in the central nervous system (CNS) [4] and ischemia-induced cardiac arrhythmias. Furthermore, DMassociated hypoglycemia can subject a person to cardiac arrhythmias, especially with type 2 diabetes (DM2) [5]. DM also increases the risk of cardiovascular disease two- to fivefold [6], which increases the risk of a pilot to become suddenly incapacitated due to acute myocardial infarction (AMI) or cardiac arrhythmia induced by AMI. In addition to coronary atherosclerosis being more common in diabetic individuals, the prognosis of diabetics with coronary atherosclerosis is poorer than in non-diabetics [7, 8]. In fact, a person with diagnosed DM2 without previous AMI has a similar risk of developing AMI than a non-diabetic with previous AMI [9]. Finally, DM also directly increases the risk of a stroke [10].

Incapacitation may also occur gradually due to hyperglycemia. In hyperglycemia, incapacitation occurs via glucagon induction and subsequent rise in blood glucose level, as well as fatty acid release from adipose tissue. If glucose balance is not corrected, this process eventually leads to diabetic ketoacidosis (DKA). Sudden pilot incapacitation due to hypoglycemia is difficult to demonstrate, since reliable blood glucose level or insulin measurement postmortem (PM) is challenging; but gradual incapacitation caused by DKA can be readily addressed [11].

Hypoglycemia, as a possible cause of pilot incapacitation, is considered in flight accidents involving pilots with DM, but the task is hampered by rapid depletion of glucose (and insulin) PM [12]. A pilot's medical history available for accident investigators is often incomplete, but by combining data from a pilot's medical records and PM toxicological and biochemical analyses estimations can be made of the short- and longterm glucose balance of DM treatment prior to an accident. In aviation fatalities biochemical PM investigation should include the analysis of long-term blood glucose balance (per cent of glycated hemoglobin, HbA1c) as well as urine and vitreous glucose levels [12-14]. New tools for investigating DM-related $\mathrm{pH}$ imbalance, for example ketone bodies, have also been added to PM investigations [11, 15], while on the basis of methodological development [16] and systematic case evaluation adding $\mathrm{HbA} 1 \mathrm{c}$ measurement into all routine PM evaluations of carbohydrate metabolism disturbance was recently suggested [16].

In this study, we analyze how DM was addressed in fatal flight accident final reports in the USA between 2011 and 2016 to better understand the challenges posed by DMrelated conditions (hypoglycemia, hyperglycemia, DKA, and microvascular diseases).

\section{Materials and methods}

Systematic analysis of fatal flight accidents in the USA between 1/1/2011-14/7/2017 with DM as an underlining factor for the incidents was performed on August 15, 2017 in the National Transportation Safety Board Aviation (NTSB) database (https://www.ntsb.gov/_layouts/ntsb.aviation/index. aspx). The search word used was "diabetes". The search returned 45 cases of 1491 fatal flight accidents during this period, of which 2 were not linked to DM (one case included discussion on management of DM after stroke and the other one discussed treatment of diabetes insipidus). PM reports were reviewed with special focus on analysis of body fluid glucose and/or HbAlc as a read-out for imbalance of DM treatment. In addition, whether DM was reported to the Federal Aviation Administration (FAA) in the latest aeromedical assessment(s) before the accident flight was assessed.

\section{Results}

\section{Anamnestic evidence of DM in the accident reports}

Of the 43 cases identified, FAA was informed in 18 (42\%) cases that the pilot had DM, and in the latest aeromedical assessment, 17 (40\%) of the cases DM were not reported. In the remaining eight (19\%) cases, it was unclear if FAA was aware of pilots' DM (Table 1) (N.B. the percent of cases, $42 \%+40 \%+19 \%$, is $101 \%$ due to the percent round up of numbers). Interestingly, in $37(86 \%)$ cases, there was

Table 1 Anamnestic knowledge of DM diagnosis of pilots by primary health care (GP) or FAA in 43 fatal flight accidents involving pilots with DM in the USA between 2011 and 2016. (N.B. the percent of cases, $42 \%+40 \%+19 \%$, is $101 \%$ due to the percent round up of numbers)

\begin{tabular}{lcc}
\hline Knowledge of DM & GP no. of cases (\%) & FAA no. of cases (\%) \\
\hline Informed & $37(86)$ & $18(42)$ \\
Not informed & $2(5)$ & $17(40)$ \\
Not known & $4(9)$ & $8(19)$ \\
\hline
\end{tabular}


indication that the general practitioner (GP) or primary care doctor was aware of the DM diagnosis, in four (9\%) cases, it was unclear if the primary health care provider was aware of the DM and in two cases, the primary care doctor was not aware of the DM diagnosis.

\section{Postmortem laboratory data}

\section{Toxicology reports}

The toxicology reports of the accident investigation reports were reviewed with special emphasis on evidence of DM. In $14(33 \%)$ of the 43 cases, PM toxicology provided evidence of $\mathrm{DM}$, i.e., glipizide alone $(n=5)$, pioglitazone alone $(n=5)$, glyburide $(n=1)$, sitagliptin $(n=2)$, combination of glipizide and pioglitazone $(n=1)$. The general characteristics of the accidents in these 14 cases are shown in Table 2. Of these 14 cases, diagnosis of DM was reported to FAA in 9 cases, in 3 cases, known DM was not reported and in 2 cases, it was unclear whether FAA was notified of the pilots' DM.

The toxicology reports indicated that many of the pilots used other personal medications, and in some cases, they used illicit drugs or medications not allowed in flying. For other PM toxicological analysis, carbon monoxide (CO) was analyzed in 15 cases (all cases were negative) and not analyzed in 28 cases. Ethanol was analyzed in 27 cases (20 negative). Six postmortem toxicology tests for ethanol were positive, but in these cases, ethanol was from sources other than ingestion. In one case, it was impossible to deduce whether the ethanol was from ingestion or other sources. Of the 16 cases where ethanol was not analyzed PM, seven cases had elevated PM glucose values. In addition, five of these 16 cases had antidiabetic medication in PM toxicology.

Table 2 Summary of postmortem-identified antidiabetic medications of pilots in DM diabetes-related fatal flight accidents in the USA between 2011 and 2016. Altogether, 43 accidents were found; in 14 cases, postmortem toxicology of the pilots was positive for antidiabetic medication. Number of lives lost in these accidents is indicated. $M$, male; No, number

\section{Glucose analysis}

Since PM glucose level analysis in urine samples has been in routine use in aviation accident investigations in the USA since 1998 or for long-term glucose balance analysis (HbAlc) since mid-2001 [17], the accident investigation final reports between 2011 and 2016 were reviewed to analyze how systematic was PM evaluation of glucose balance (Table 3). Quantitative information on any type of glucose information (vitreous, blood, urine) was reported in only 11 cases; in one report urine glucose was "detected" but not quantitated. Average percent of blood HbA1c $(n=9)$ was $7.9 \%(4.8$ $10.3 \%)$, average urine glucose $(n=11)$ was $1271 \mathrm{mg} / \mathrm{dl}(12$ $3000 \mathrm{mg} / \mathrm{dl})$ and average vitreous glucose $(n=8)$ was $42 \mathrm{mg} /$ dl $(0-149 \mathrm{mg} / \mathrm{dl})$. No information on possible ketone body measurements was available. Two hundred thirty-four milligram per deciliter [18] or $180 \mathrm{mg} / \mathrm{dl}$ [19] have been suggested as cutoff values for fatal antemortem (AM) hyperglycemia in vitreous fluid. Practically, any trace of glucose in the vitreous fluid is likely an indication of antermortem hyperglycemia [20]. For urine PM glucose measurements, specifying cutoff values for evaluating AM glucose balance from PM urine samples is challenging, and the urine glucose measurement should primarily be used to confirm vitreous findings [21-23].

\section{Discussion}

Between 2011 and 2016, 43 flight accidents related to DM in the USA were discovered in the NTSB aviation database (out of 1491 accidents; 3\%). As a reference, Chaturvedi et al. performed a search of elevated vitreous and/or urine glucose in fatal flight accidents between 1998 and 2005 in the USA for 2487 deceased pilots, and they found 43 had elevated glucose

\begin{tabular}{lll}
\hline DM medication in toxicology & Pilot age, sex, and accident year & No. of lost lives in the accident \\
\hline Glipizide & $60 \mathrm{M}, 2011$ & 1 \\
$65 \mathrm{M}, 2011$ & 1 \\
$56 \mathrm{M}, 2012$ & 1 \\
$69 \mathrm{M}, 2012$ & 1 \\
$68 \mathrm{M}, 2016$ & 1 \\
& $53 \mathrm{M}, 2011$ & 1 \\
Pioglitazone & $63 \mathrm{M} 2012$ & 1 \\
& $62 \mathrm{M}, 2012$ & 1 \\
& $74 \mathrm{M}, 2013$ & 1 \\
& $44 \mathrm{M}, 2013$ & 1 \\
Glyburide & $73 \mathrm{M}, 2014$ & 2 \\
Sitagliptin & $63 \mathrm{M}, 2015^{*}$ & 1 \\
Glipizide + pioglitazone & $56 \mathrm{M}, 2016$ & 1 \\
\hline
\end{tabular}

*flight student, **flight instrutor 
Table 3 Available postmortem glucose metabolism measurements from diabetes DMrelated flight accidents between 2011 and 2016 in the USA. Where available, the source of the blood sample for HbA1c measurement is indicated in parentheses. $N / A$ data not available, $M$ male, Blood source: $C$ cavity, $H$ heart, $F$ femoral artery

\begin{tabular}{llll}
\hline Pilot age, sex, and accident year & Blood Hb1Ac $\%$ & Urine glucose (mg/dl) & Vitreous glucose (mg/dl) \\
\hline $63 \mathrm{M}, 2011$ & $4.8 \%(\mathrm{C})$ & 3000 & Not detected \\
$63 \mathrm{M}, 2012$ & N/A & Detected & Not detected \\
$51 \mathrm{M}, 2012$ & N/A & 2460 & N/A \\
$79 \mathrm{M}, 2012 * *$ & $8.7 \%$ & 1150 & N/A \\
$58 \mathrm{M}, 2013$ & $7.0 \%(\mathrm{H})$ & 1949 & 149 \\
$58 \mathrm{M}, 2013$ & $9.2 \%(\mathrm{C})$ & 484 & N/A \\
$51 \mathrm{M}, 2013$ & $10.3 \%(\mathrm{C})$ & 1668 & 46 \\
$56 \mathrm{M}, 2013$ & $8.6 \% .(\mathrm{F})$ & 2514 & 82 \\
$73 \mathrm{M}, 2014$ & $6.1 \%$ & 12 & N/A \\
$47 \mathrm{M}, 2014$ & N/A & 248 & 51 \\
$57 \mathrm{M}, 2014$ & $7.4 \%$ & 135 & Not detected \\
$80 \mathrm{M}, 2015$ & $9.0 \%$ & 366 & 8 \\
\hline
\end{tabular}

**flight instructor

levels (1.7\%) [17]. Altogether, 58 lives were lost in the reviewed accidents here; the mean age of pilots (all male) was 62.4 (range 28-83 years), the median age was 63 years. In addition, in these accidents eight persons were severely injured, and one person suffered minor injuries. In 25 cases, health-related issues were not indicated as affecting the accident according to the accident final report; while in 18 cases, health of the pilot was either contributing to the accident or it was not possible to rule out that a health issue contributed to the accident. Autopsies revealed that in nine cases, the pilots had coronary atherosclerosis. In cases, where coronary atherosclerotic occlusion (\%) was indicated in the autopsy report $(n=7)$, the area of coronary artery occlusion was $>75 \%$. In other cases, the occlusion was indicated by description as "severe" $(n=1)$ or "mild" $(n=1)$. In six additional cases, atherosclerosis was present, but either its severity was not reported in the autopsy or the information was based on medical records.

Fatal flight accidents are investigated with a standardized protocol (ICAO annex 13) [24]. The protocol aims at investigating all contributory explanations for the accident and to identify the actual cause for an accident so that future accidents may be prevented. The medical condition of a pilot is always a critical part of such an investigation. With the increasing incidence of DM2, its role as a possible cause or contributing factor in an accident needs to be considered. In 2013, global prevalence of DM (both DM1 and DM2) was estimated to be 382 million people and in 2035, it is estimated to be 592 million people, with DM2 representing $\sim 85 \%$ of all DM cases [25]. Both the risk for AMI and for stroke are elevated in diabetic individuals $[6,9,10]$. In addition, older individuals with DM are at substantial risk of acute cardiovascular complications [26], which underlines the importance of health reviews of aging pilots especially those with DM.

The role of insulin-treated DM is relatively low as a cause of accidents involving pilots carrying a third-class medical certificate, a certificate required to obtain a private pilot license in the USA [2]. This estimation is based on the analysis of 1500 waivers for insulin-treated DM approved by FAA, which did not indicate increased risk for fatal accidents between 1997 and 2014 for pilots with DM [2]. The overall accident rate for pilots with a waiver for insulin was 7.0 for 100,000 flight hours, while an estimate for all third-class pilots was exactly the same (7.0/100000 flight hours) [2]. This finding would suggest that insulin-treated DM may not add risk for an accident. However, this conclusion may underestimate the role of DM, due to the fact that hypoglycemia that might affect the accident is difficult to address in PM diagnostics. Interestingly, the prevalence of DM (DM1 and DM2) in the USA is $9.4 \%$ (http://www.diabetes.org/diabetes-basics/ statistics/) while pilots with DM in fatal flight accidents in the USA the prevalence was $\sim 3 \%$, which would also suggest that DM was not increasing the risk for fatal accidents since prevalence among diabetic pilots in accidents is actually lower than DM in the general population. Various explanations could account for this difference. First, the lack of information flow of the pilots' DM to the aviation authority (FAA) may result in an underestimation of the number of diabetic pilots (Table 1) and thus could discourage the search for DM in accident review. Retrospectively, in cases studied herein, FAA was informed in only $43 \%$ of the cases that the pilots had DM. Second, medical screening of pilots may remove diabetics with more severe DM-related complications as pilot candidates. Third, the medical screening (for Hb1 Ac and diabetic complications) combined with more regular doctor visits for pilots with DM might select pilots with DM that are overall more healthier than non-diabetic pilots. Fourth, the difficulty in measuring postmortem hyperglycemia and particularly hypoglycemia might underestimate the number of diabetes-related accidents. Fifth, a combination of these factors could be involved. 
Interestingly, antidiabetic medication in the toxicology reports and elevated glucose in the body fluids correlated only in two cases. This could be due to a good balance of DM treatment. Those individuals not using the DM medication (either for not knowing they did have DM or for lack of compliance) would have elevated glucose levels. However, it is also possible that antidiabetic medication subjects one to hypoglycemia. Another common cause of hypoglycemia could be ethanol particularly combined with antidiabetic medication [27]. In this study, of the 16 cases where ethanol was not analyzed PM, seven cases had elevated PM glucose values which leaves open the theoretical possibility of ethanol-induced hypoglycemia in nine cases. One of these individuals with unclear ethanol status and antidiabetic medication had elevated glucose levels PM. In four cases with antidiabetic medication, PM without ethanol assessment hypoglycemia status remain unknown, but possible.

In a diabetic individual glucose, $\mathrm{HbA} 1 \mathrm{c} \%$ and ketone body levels are measured to determine the balance of DM treatment. In clinics, blood (and urine) glucose levels can be easily measured. However, when treating diabetic patients, long-term glucose levels are important for patient evaluation [28]. $\mathrm{HbA} 1 \mathrm{c} \%$ provides a reliable measure of blood glucose level over a period of several weeks (4-8 weeks), while it does not catch acute hypoglycemia. In clinics, $\mathrm{HbA} 1 \mathrm{c}$ measurement is usually combined with a measurement of blood glucose level (either fasting or non-fasting) as well as serum ketone bodies and acidosis (serum bicarbonate) $[4,28]$. In the routine postmortem investigation, DM as a primary or underlining cause of death is difficult to diagnose due to lack of specific macroscopic and microscopic findings. Also, PM glucose analysis is not feasible due to rapid glucose metabolization (glycolysis) into lactate [21]. However, using the recently developed biochemical analyses, and alternative matrices (such as vitreous humor and urine), fatal DM-related ketoacidosis is readily diagnosed, even in cases of no medical history of DM or cadavers with advanced postmortem changes [22, 29, 30]. Recently Palmiere discussed the caveats on using a single measurement as a definitive indication of dysregulation of glucose metabolism [31]; single measurement of glucose metabolism from any PM sample is likely unreliable. HBA1c and vitreous glucose measurements are used to assess glucose balance. The measurement of urine glucose can be supportive of the results obtained in vitreous fluid but if the urine is the only sample available, the significance of the measurement results should be carefully estimated [12-14]. It is clear that more sophisticated measurement tools of glucose balance will help in analyzing AM glucose balance in postmortem analysis; one candidate approach could be 1,5anhydro-d-glucitol, which has shown promise in identifying AM diabetic coma in postmortem analysis [32]. In the USA, vitreous fluid and urine glucose analyses were implemented as routine tests for flight accident investigations in the beginning of 1998 , and blood HbAlc testing was implemented in the middle of 2001 [17]. One difficulty in assessing DM as a cause of pilot incapacitation in aviation accidents is the high energy forces associated with the accidents which make it difficult to obtain samples (body fluids) for analysis. Another critical feature in PM investigation related to DM is the status of coronary arteries and major CNS-arteries in context of atherosclerosis as a complication of DM. Finally, an accident investigation needs to take into account what factor(s) medication(s) may play in pilot incapacitation. Insulin and oral DM medication are a risk factor for hypoglycemia, and other simultaneous medications via various mechanisms may increase the hypoglycemic effect of oral DM medication [33]. Thus, pilots' medical records for prescriptive or over the counter medications, as well as toxicological report findings, need to be reviewed from the perspective of medication-induced hypoglycemia. Recently, analysis of exogenous insulin has become possible from postmortem samples [30, 31, 34, 35] which can also be added to aviation fatality investigation when hypoglycemia due to insulin use is suspected.

Multidrug use is a common finding in the realm of medicolegal autopsies [36, 37] and it is also challenge for fatal aviation accident investigations, as well as to AMEs and pilots' general practitioners. In cases reviewed herein, the median age of pilots during the accident was 63 . Recently, $13 \%$ of US general aviation pilots over 70 years involved in a fatal accident were shown to have used three or more medications during the accident as indicated by the toxicology findings, with antihistamines and antidepressants the most frequently discovered substances [3].

Hypoglycemia, as a cause of sudden pilot incapacitation, is currently practically nearly impossible to address. Hyperglycemia and insulin deficiency-related DKA as cause of gradual pilot incapacitation should be investigated more extensively. Overall, given the high prevalence of DM, especially in the aging population, and the lack of disclosure of DM to FAA indicated in the study herein, glucose measurements, accompanied by measurement of ketone bodies (acetone, acetoacetate, $\beta$-hydroxybutyrate), should be a part of a standard investigation in an aviation fatality during the postmortem investigation of the pilot.

Funding information The work was funded by Academy of Finland (ISJ, projects 25013080481 and 25013142041), Finnish Medical Foundation (ISJ), Tampere Tuberculosis Foundation (ISJ), and Sigrid Juselius Foundation (ISJ).

\section{Compliance with ethical standards}

Conflict of interest The authors declare that they have no conflict of interest. 


\section{References}

1. Mitchell SJ, Hine J, Vening J, Montague J, Evans S, Shaw KM, Frier BM, Heller SR, Russell-Jones DL (2017) A UK civil aviation authority protocol to allow pilots with insulin-treated diabetes to fly commercial aircraft. Lancet Diabetes Endocrinol 5:677-679

2. Mills WD, DeJohn CA, Alaziz M (2017) The U.S. experience with waivers for insulin-treated pilots. Aerosp Med Hum Perform 88: 34-41

3. Vuorio A, Asmayawati S, Budowle B, Griffiths R, Strandberg T, Kuoppala J, Sajantila A (2017) General aviation pilots over 70 years old. Aerosp Med Hum Perform 88:142-145

4. Fazeli Farsani S, Brodovicz K, Soleymanlou N, Marquard J, Wissinger E, Maiese BA (2017) Incidence and prevalence of diabetic ketoacidosis (DKA) among adults with type 1 diabetes mellitus (T1D): a systematic literature review. BMJ Open 7: e016587

5. Chow E, Bernjak A, Williams S, Fawdry RA, Hibbert S, Freeman J, Sheridan PJ, Heller SR (2014) Risk of cardiac arrhythmias during hypoglycemia in patients with type 2 diabetes and cardiovascular risk. Diabetes 63:1738-1747

6. Preis SR, Hwang SJ, Coady S, Pencina MJ, D'Agostino RBS, Savage PJ, Levy D, Fox CS (2009) Trends in all-cause and cardiovascular disease mortality among women and men with and without diabetes mellitus in the Framingham Heart Study, 1950 to 2005. Circulation 119:1728-1735

7. Grundy SM, Balady GJ, Criqui MH, Fletcher G, Greenland P, Hiratzka LF, Houston-Miller N, Kris-Etherton P, Krumholz HM, LaRosa J, Ockene IS, Pearson TA, Reed J, Washington R, Smith SC, Jr (1998) Primary prevention of coronary heart disease: guidance from Framingham: a statement for healthcare professionals from the AHA Task Force on Risk Reduction. American Heart Association. Circulation 97:1876-1887

8. Huxley R, Barzi F, Woodward M (2006) Excess risk of fatal coronary heart disease associated with diabetes in men and women: meta-analysis of 37 prospective cohort studies. BMJ 332:73-78

9. Haffner SM, Lehto S, Ronnemaa T, Pyorala K, Laakso M (1998) Mortality from coronary heart disease in subjects with type 2 diabetes and in nondiabetic subjects with and without prior myocardial infarction. N Engl J Med 339:229-234

10. Chen R, Ovbiagele B, Feng W (2016) Diabetes and stroke: epidemiology, pathophysiology, pharmaceuticals and outcomes. Am J Med Sci 351:380-386

11. Walta AM, Keltanen T, Lindroos K, Sajantila A (2016) The usefulness of point-of-care (POC) tests in screening elevated glucose and ketone body levels postmortem. Forensic Sci Int 266:299-303

12. Khuu HM, Robinson CA, Brissie RM, Konrad RJ (1999) Postmortem diagnosis of unsuspected diabetes mellitus established by determination of decedent's hemoglobin A1c level. J Forensic Sci 44:643-646

13. Canfield DV, Chaturvedi AK, Boren HK, Veronneau SJ, White VL (2001) Abnormal glucose levels found in transportation accidents. Aviat Space Environ Med 72:813-815

14. Chaturvedi AK, Smith DR, Soper JW, Canfield DV, Whinnery JE (2003) Characteristics and toxicological processing of postmortem pilot specimens* from fatal civil aviation accidents. Aviat Space Environ Med 74:252-259

15. Keltanen T, Sajantila A, Palo JU, Partanen T, Valonen T, Lindroos K (2013) Assessment of Traub formula and ketone bodies in cause of death investigations. Int J Legal Med 127:1131-1137

16. Keltanen T, Sajantila A, Valonen T, Vanhala T, Lindroos K (2013) Measuring postmortem glycated hemoglobin - a comparison of three methods. Leg Med (Tokyo) 15:72-78
17. Chaturvedi AK, Botch SR, Canfield DV, Forster EM (2009) Vitreous fluid and/or urine glucose concentrations in 1335 civil aviation accident pilot fatalities. J Forensic Sci 54:715-720

18. Karlovsek MZ (2004) Diagnostic values of combined glucose and lactate values in cerebrospinal fluid and vitreous humour-our experiences. Forensic Sci Int 146(Suppl):S19-S23

19. Zilg B, Alkass K, Berg S, Druid H (2009) Postmortem identification of hyperglycemia. Forensic Sci Int 185:89-95

20. Belsey SL, Flanagan RJ (2016) Postmortem biochemistry: current applications. J Forensic Legal Med 41:49-57

21. Hess C, Musshoff F, Madea B (2011) Disorders of glucose metabolism-post mortem analyses in forensic cases: part I. Int J Legal Med 125:163-170

22. Palmiere C, Lesta Mdel M, Sabatasso S, Mangin P, Augsburger M, Sporkert F (2012) Usefulness of postmortem biochemistry in forensic pathology: illustrative case reports. Leg Med (Tokyo) 14:27-35

23. Hess C, Wollner K, Musshoff F, Madea B (2013) Detection of diabetic metabolism disorders post-mortem - forensic case reports on cause of death hyperglycaemia. Drug Test Anal 5:795-801

24. Rasmussen J, Svedung I (2000) Proactive risk management in a dynamic society. Swedish Rescue Services Agency, Karlstad

25. Forouhi NG, Wareham NJ (2014) Epidemiology of diabetes. Medicine (Abingdon) 42:698-702

26. Kirkman MS, Briscoe VJ, Clark N, Florez H, Haas LB, Halter JB, Huang ES, Korytkowski MT, Munshi MN, Odegard PS, Pratley RE, Swift CS (2012) Diabetes in older adults. Diabetes Care 35: 2650-2664

27. Kugelberg FC, Jones AW (2007) Interpreting results of ethanol analysis in postmortem specimens: a review of the literature. Forensic Sci Int 165:10-29

28. Nathan DM (2015) Diabetes: advances in diagnosis and treatment. JAMA 314:1052-1062

29. Palmiere C, Bardy D, Mangin P, Werner D (2013) Postmortem diagnosis of unsuspected diabetes mellitus. Forensic Sci Int 226: 160-167

30. Hess C, Madea B, Daldrup T, Musshoff F (2013) Determination of hypoglycaemia induced by insulin or its synthetic analogues post mortem. Drug Test Anal 5:802-807

31. Palmiere C (2015) Postmortem diagnosis of diabetes mellitus and its complications. Croat Med J 56:181-193

32. Sydow K, Wiedfeld C, Musshoff F, Madea B, Tschoepe D, Stratmann B, Hess C (2018) Evaluation of 1,5-anhydro-d-glucitol in clinical and forensic urine samples. Forensic Sci Int 287:88-97

33. Amiel SA, Dixon T, Mann R, Jameson K (2008) Hypoglycaemia in type 2 diabetes. Diabet Med 25:245-254

34. Thevis M, Thomas A, Schanzer W, Ostman P, Ojanpera I (2012) Measuring insulin in human vitreous humour using LC-MS/MS. Drug Test Anal 4:53-56

35. Ojanpera I, Sajantila A, Vinogradova L, Thomas A, Schanzer W, Thevis M (2013) Post-mortem vitreous humour as potential specimen for detection of insulin analogues by LC-MS/MS. Forensic Sci Int 233:328-332

36. Jones AW, Kugelberg FC, Holmgren A, Ahlner J (2011) Drug poisoning deaths in Sweden show a predominance of ethanol in mono-intoxications, adverse drug-alcohol interactions and polydrug use. Forensic Sci Int 206:43-51

37. Jones AW, Holmgren A, Ahlner J (2016) Post-mortem concentrations of drugs determined in femoral blood in single-drug fatalities compared with multi-drug poisoning deaths. Forensic Sci Int 267: 96-103 